\title{
Amalgamated Generalized Low Density Parity Check and Luby Transform Codes for the Wireless Internet
}

\author{
T. D. Nguyen, FC. Kuo, L. L. Yang and ${ }^{1}$ L. Hanzo \\ School of ECS, University of Southampton, SO17 1BJ, UK. \\ Tel: +44-23-8059 3125, Fax: +44-23-8059 4508 \\ Email: ${ }^{1}$ lh@ecs.soton.ac.uk, http://www-mobile.ecs.soton.ac.uk
}

\begin{abstract}
Luby Transform codes (LT) were originally designed for the Binary Erasure Channel (BEC) encountered owing to randomly dropped packets in the statistical multiplexing aided Internet, where transmitted packets are not affected by the fading or noise of the environment. For the sake of transmitting data over the BEC routinely encountered in statistical multiplexing aided wireless Internet-style scenarios, LT codes have to be combined with classic channel codes. In this paper we introduce a novel almagamated LT coding and Generalized Low Density Parity Check (G-LDPC) coding scheme. Upon exploiting the packet erasure information provided by the GLDPC decoder for the LT decoder, the proposed scheme achieves a low Bit Error Ratio (BER) for Packet Error Ratio (PER) $E_{b} / N_{0}$ values in excess of 3.2dB when transmitting data over the AWGN-BEC channel having a packet erasure probability of $P_{e}=\mathbf{0 . 1}$. When communicating over the Rayleigh-BEC channel at $P_{e}=\mathbf{0 . 1}$, an $E_{b} / N_{0}$ value of 5.3 dB was required. In comparison to the serially concatenated LT-GLDPC scheme using no information exchange between the G-LDPC decoder and the LT decoder, the amalgamated LT-GLDPC arrangement achieved an $E_{b} / N_{0}$ gain of up to 1.6-2.5 dB .
\end{abstract}

\section{INTRODUCTION}

Generalized Low Density Parity Check (G-LDPC) codes [1] [2] offer the beneficial design option of replacing the simple parity check codes of classic LDPC codes by more sophisticated constituent encoders, such as for example binary or nonbinary Bose-Chaudhuri-Hocquenghem (BCH) codes [3]. They also benefit from innovatively combining low-complexity constituent encoders and decoders with intelligent iterative detection in the interest of approaching the attainable capacity at a moderate complexity. In this treatise GLDPC codes are amalgamated with Luby Transform (LT) codes [4], which were originally designed for achieving an infinitesimally low packet error ratio (PER) over the Binary Erasure Channel (BEC) routinely encountered in statistical multiplexing aided wireless Internet-style scenarios. However, LT codes were not intended for communications over gravely error-infested hostile channels encountered in the wireless Internet. Hence, the novel con- tribution of this paper is that we amalgamated LT codes with GLDPC codes, where the latter combats the effects of channel errors and informs the LT decoder, as to when it is unsafe to carry out LT decoding without catastrophic error propagation across the LT-encoded packets. We will demonstrate that as a result, $E_{b} / N_{0}$ gains of $1.6-2.5 \mathrm{~dB}$ are achievable in the Rayleigh-faded wireless Internet environment considered. In fact, owing to their potentially avalanche-like decoding error propagation across LT-encoded packets, the employment of LT codes may catastrophically degrade the overall system performance, unless they are combined with powerful Forward Error Connection (FEC) codes. As a benchmarker, we used the powerful combination of an iteratively detected Bit Interleaved Coded Modulation and LT coding scheme [5]. However, the operation of the BICM-ID and LT schemes in [5] was based on simply passing the channel-decoded bits to the LT decoder, rather than on intrinsically amalgamating their operation. Any potential residual errors persisting at the output of the BICM-ID decoder were passed to the LT decoder, which may have precipitated the errors by propagating them to further LT-encoded packets. Hence, in this treatise LT codes are intrinsically amalgamated with GLDPC codes [1] [2] [6]. More explicitly, before LT decoding ensues, each GLDPC codeword is tested by the classic parity check of $X \cdot H^{T}=0$, to ensure that a legitimate although not necessarily error-free codeword was generated, where $X$ represents a legitimate GLDPC codeword, while $H^{T}$ is the transpose of the parity check matrix. This measure prevents the LT decoder from propagating GLDPC decoding errors from one LT-encoded packet to another, unless the GLDPC decoder failed to recognize that a legitimate, but erroneously decoded codeword was encountered.

In Section 2 we describe the proposed GLDPC-LT scheme, while in Section 3 we characterise its achievable performance. Finally, in Section 4 we offer our conclusions.

\section{AMALGAMATED LT AND GLDPC CODING}

The schematic of the proposed coding arrangement is shown in Fig. 1, while the mapping of the GLDPC codewords to an LTencoded transmitted packet is seen in the Fig. 3. To elaborate a little further, the philosophy of GLDPC codes is reminiscent of the classic turbo encoding principle of using low-complexity 
constituent encoders and decoders for creating powerful, nearcapacity iterative decoding schemes having a moderate complexity. The liaison of the GLDPC decoder with the LT decoder is based on counting the number of illegitimate GLDPC codewords in an LT-encoded packet and then comparing $E$ to an appropriately chosen threshold $T$. To elaborate a little further, the decoding operations commence by setting the threshold $T$ to zero, indicating that only error-free GLDPC packets are allowed to be passed to the LT decoder, in order to avoid LT-decoding-induced inter-packet error propagation. If we have $E=0$, no erroneous GLDPC codewords were found within the current LT-encoded packet and hence this packet is ready for LT decoding. If this error free LT-packet is a degreeone packet, LT decoding may commence immediately. By contrast, if no degree-one packet is available at the LT decoder, the commencement of the LT-decoding process is defened until the arrival of a degree-one packet. For the sake of avoiding the risk of losing all the remaining undecoded source packets, in the absence of a degree-one packet, we may decide to increase the threshold $T$, in order to request further LT-encoded packets, which now contains $E>0$ number of erroneous GLDPC codewords. If this is the case, the residual error of the current LT-encoded packets are passed on to other packets in the same bit position owing to their modulo-2 relationship. To elaborate a little further, it is determined by the degree of the packet concerned, how many further errors are caused. Therefore erroneous GLDPC codewords should only by considered for LT decoding, if they belong to degree-one packets, since the absence of an error-free degree one packet results in losing all the remaining packets. This measure prevents the propagation of GLDPC decoding errors to other LT packets, since degreeone packets are decoded without the aid of other packets, while avoiding the risk of losing all the remaining packets owing to the absence of degree-one packets.

By contrast, in case of GLDPC decoding errors in LT packets having a higher degree the erasure of the packet does not prevent decoding the rest of the packets, but would propagate the errors to as many LT-encoded packets, as the degree of the packet concerned. Hence, higher-degree packets containing GLDPC decoding errors have to be considered as erased. However, given the ability of the LT decoder to fill the erased codewords, the amalgamated scheme is expected to outperform both its components at a modest complexity. For simplicity, we refer to the system benefiting from information exchange between the LT decoder and the GLDPC decoder as Scheme 1, while the one dispensing with it is termed as Scheme 2.

\section{SYSTEM PARAMETERS AND PERFORMANCE}

The parameters of the system investigated are summarized in Table 1. The GLDPC encoder used the binary $\operatorname{BCH}(n, k, t)=$ $\mathrm{BCH}(15,11,1)$ constituent codes, which operated over the Galois Field GF(16) and each BCH codeword was capable of correcting $t=1$ error, when using classic algebraic decoding [3].

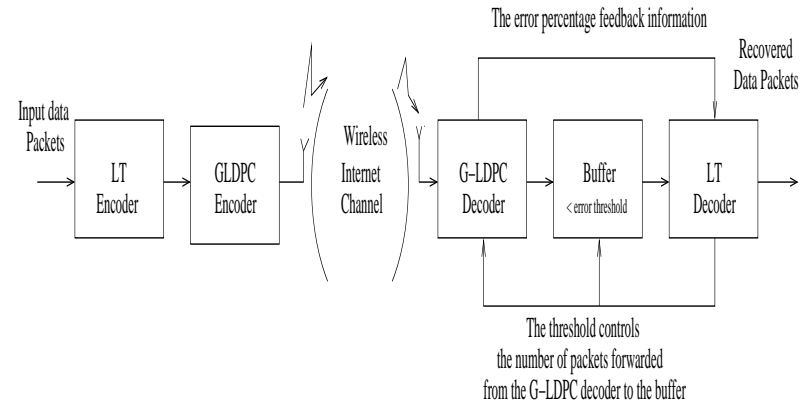

Figure 1: Amalgamated GLDPC codes and LT codes

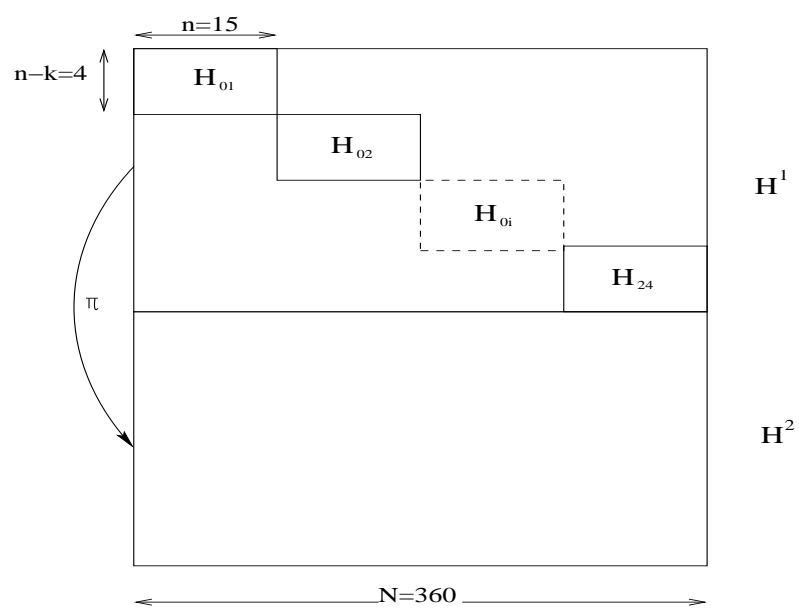

Figure 2: The structure of the GLDPC parity check matrix

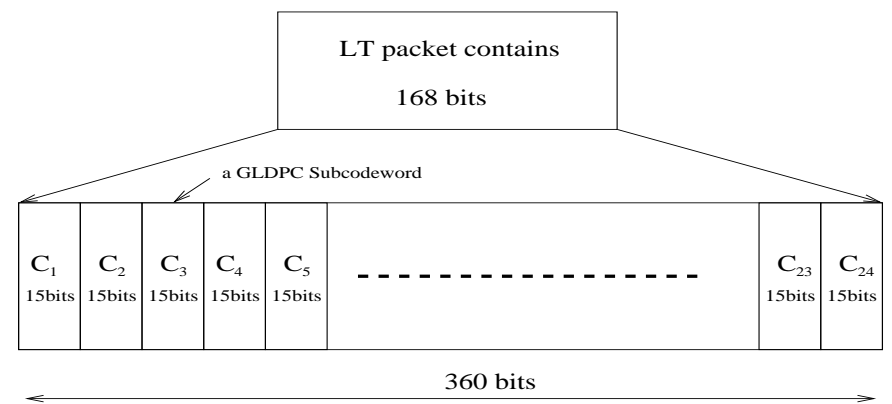

Figure 3: The relation between the LT-encoded packet and the G-LDPC codewords

\begin{tabular}{|l|r|}
\hline Erasure probability & $P_{e}=0.1$ \\
LT code parameters & $\delta=0.5 \mathrm{c}=0.1$ \\
Number of bits per LT packet & 168 \\
Number of source packets & 10000 \\
Number of encoded LT packets & 13000 \\
GLDPC component codes & $\mathrm{BCH}(15,11,1)$ \\
Component code rate of GLDPC & $r=\frac{11}{15}$ \\
Code rate of GLDPC & $\frac{7}{15}$ \\
Modulation & BPSK \\
\hline
\end{tabular}

Table 1: System parameters. 
The number of input data bits per LT-encoded packet was 168 and each of these packets was mapped to 24 GLDPC codewords, as seen in Fig. 3. We can calculate the number of GLDPC subcodewords in an LT-encoded packet as follows. We denote the code rate of the component $\mathrm{BCH}$ codes by $r$, while $R$ is the overall code rate of the GLDPC code. Observe in Fig. 2 that the parity matrix of the GLDPC encoder has two superblocks, namely $H_{1}$ and $H_{2}$, where $H_{2}$ represents a permuted version of $H_{1}$ as detailed in [1] [2] [6]. More specifically, $H_{1}$ contains $L=24$ parity matrices corresponding to the $\operatorname{BCH}(15,11,1)$ codes given that we use $J=2$ GLDPC superblocks and that the number of parity bits is $(n-k)=4$, the resultant GLDPC code has a totall of $4 \cdot 5=8$ parity bits hence the resultant GLDPC code becomes $R=\frac{7}{15}$. More generally, the overall code rate $R$ is given by $R=1-J \cdot\left(1-\frac{k}{n}\right)$ [2], where again, $J=2$ is the number of the so-called GLDPC superblocks and $r=\frac{k}{n}=\frac{11}{15}$, therefore we arrive at $R=\frac{7}{15}$. Hence, as seen in Fig. 3, the number of GLDPC subcodewords used by the GLDPC scheme encoding a single LT source packet equals to $\frac{168}{7}=24$. The LT encoder used the Robust Soliton Distribution (RSD) designed in [5] and imposed a 30\% packet overhead, while using the LT encoder parameters of $\delta=0.5$ and $c=0.1$ detailed in [5]. The number of GLDPC decoding iterations was set to $I=10$ in the schematic of Fig. 4. The BER performance of the system using the parameters of Table 1 is shown in Figures 5 and 6, when communicating over both AWGN and Rayleigh channels, initially inflicting no erasures, ie. when we have $P_{e}=0$. By contrast, in Fig. 7 and Fig. 8 we used a somewhat unconventional channel model, which may be encountered in AWGN-contaminated and Rayleigh-faded radio links also subjected to a statistical multiplexing-induced LT-packet erasure probability of $P_{e}=0.1$. Naturally, the packet erasure events may have been imposed by other phenomena, such as shadow-fading-infested GLDPC packets.

Observe in Fig. 7 that the amalgamated LT-GLDPC Scheme 1 exhibits an approximately $1.6 \mathrm{~dB} E_{b} / N_{0}$ gain over Scheme 2, when using the Approximate-log MAP decoder [3]. Similar performance trends were observed in Fig. 8 for transmission over uncorrelated Rayleigh channels, exhibiting an approximately $1.5 \mathrm{~dB}$ gain for Scheme 1 employing the proposed threshold-based information exchange between the GLDPC and LT decoders. Fig. 9 and 10 characterise the Packet Error Ratio (PER) performance of the amalgamated LT-GLDPC Scheme 1 as well as that of Scheme 2, when the data is transmitted over the AWGN-BEC channel at $E_{b} / N_{0}=4 \mathrm{~dB}$ employing both decoding methods, namely that benefitting from passing moderately contaminated degree-one packets to the LT decoder from the GLDPC decoder and that erasing them. We observe from Fig. 9 and Fig. 10 that the performance of Scheme 1 is better than that of Scheme 2. As seen in Fig. 9 and Fig. 10, Scheme 1 is capable of achieving error free at $P_{e}=0.16$, while the latter has a PER of about $10^{-1}$ even at $P_{e}=0.10$.

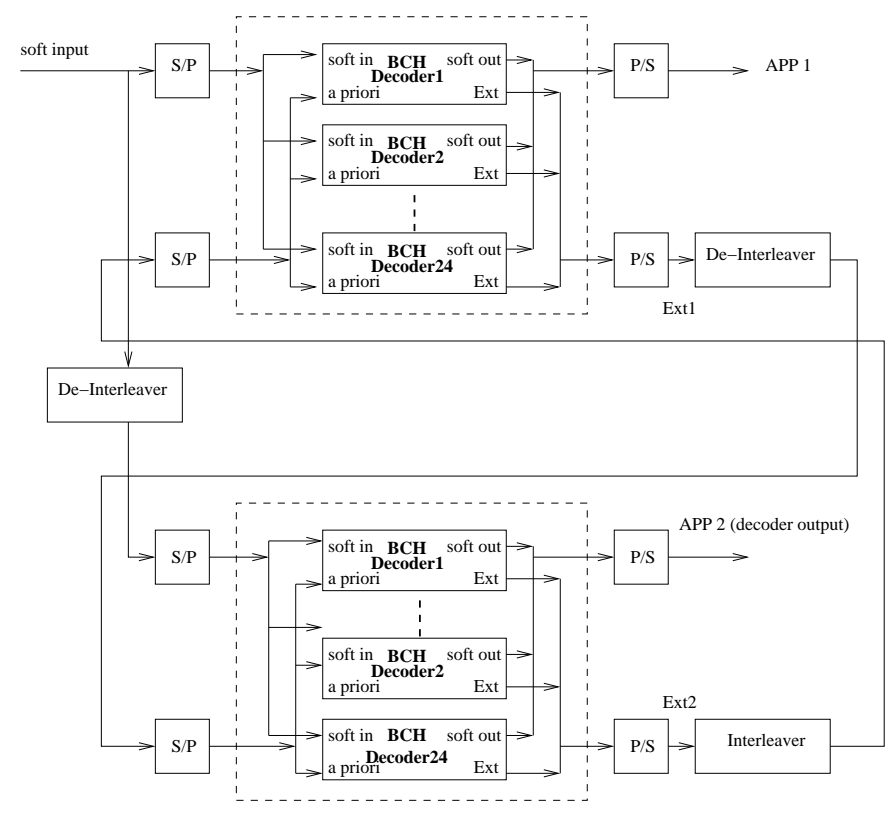

Figure 4: The GLDPC Decoder

\section{SUMMARY AND CONCLUSION}

In this contribution an amalgamated LT-GLDPC coding scheme was proposed, where the constituent decoders exchanged information. In an effort to avoid curtailing the LT decoding operation in the absence of degree-one packets, Scheme 1 allowed the GLDPC decoder to pass a maximum of $T$ illegitimate GLDPC codewords to the LT decoder. By contrast, Scheme 2 allowed the GLDPC decoder to pass all GLDPC codewords to the LT decoder, which causes the error propagation during the LT decoding operation. As a result, $E_{b} / N_{0}$ gains of 1.6 and $2.5 \mathrm{~dB}$ were achieved over the BEC-AWGN and BEC-Rayleigh channels considered.

\section{REFERENCES}

[1] M. Lentmaier, K.S. Zigangirov., "On Generalized Low-Density ParityCheck Codes Based on Hamming Component Codes," IEEE Communications Letters, pp. 248-250., Aug 1999.

[2] FC. Kuo, L. Hanzo., "Symbol-Flipping Based Decoding of Generalized Low-Density Parity-Check Codes over GF(q)," Proceedings and CDROM of IEEE WCNC 2006, 3-6 April,2006, Las Vegas, vol. 138, pp. 1207-1211, May 2006.

[3] L. Hanzo,T.H. Liew and B.L.Yeap, Turbo Coding, Turbo Equalisation and Space-Time Coding for Transmission over Fading Channels. wiley \& IEEE Press, 2002.

[4] M. Luby, "LT codes," in Proceeding of the 43rd Annual IEEE Symposium on Foundations of Computer Science, pp. 271-282, November 2002.

[5] R. Tee, T.D. Nguyen, L-L. Yang and L. Hanzo., "Serially Concatenated Luby Transform Coding and Bit-Interleaved Coded Modulation Using Iterative Decoding for the Wireless Internet," Proceedings of VTC 2006 Spring, Melbourne,CD ROM, vol. 138, pp. 177-182, May 2006.

[6] S. Hirst and B. Honary., "Decoding of generalised low-density paritycheck codes using weighted bit-flip voting," IEE Proceedings Communications, pp. 1-5, Feb 2002. 


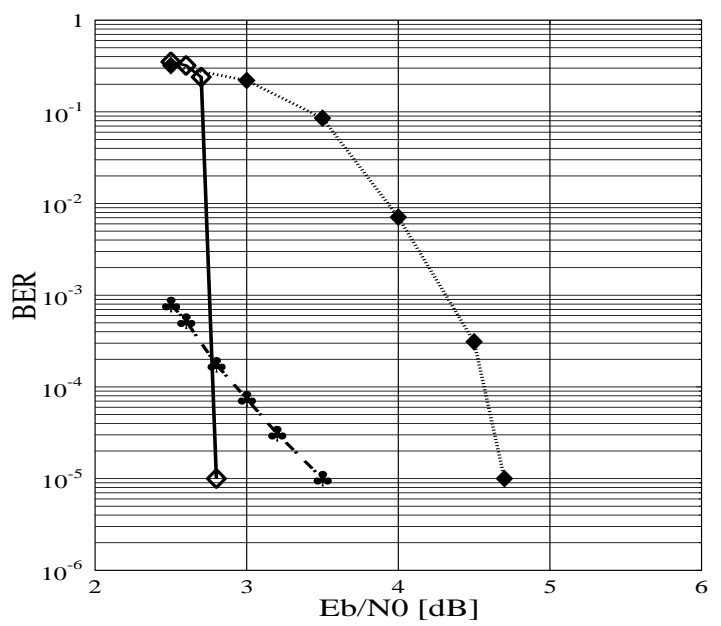

The serially concatenated LT-GLDPC,Approx-log MAP, Scheme 1

-. - GLDPC $(15,7)$ code, using Approx-log MAP decoding, No LT decoding

Figure 5: The performance of the serially concatenated LT and G-LDPC code, using BPSK modulation for transmission over the AWGN channel, $P_{e}=0$

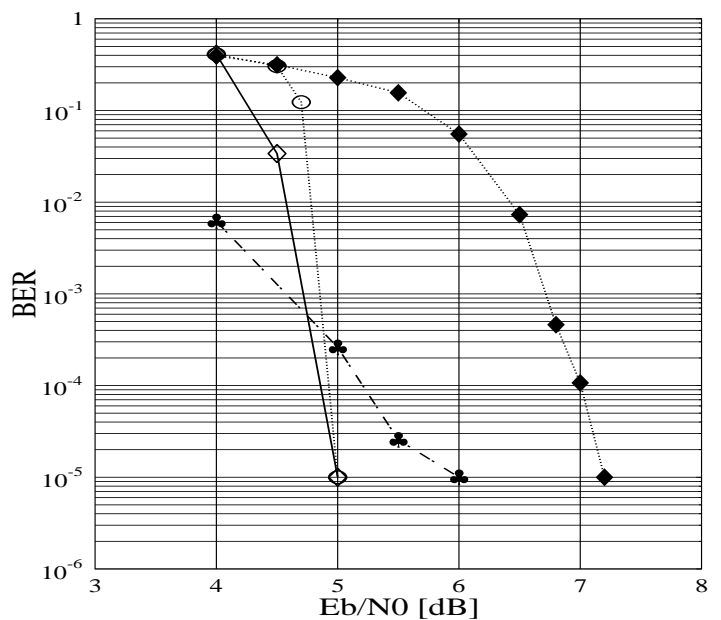

The serially concatenated LT-GLDPC,Approx-log MAP, Scheme 1

The serially concatenated LT-GLDPC,Approx-log MAP,Scheme 1 , using the threshold T=0

GLDPC $(15,7)$ code, using Approx-log MAP decoding, No LT decoding

Figure 6: The performance of the serially concatenated LT and G-LDPC code, using BPSK modulation for transmission over the Rayleigh channel, $P_{e}=0$

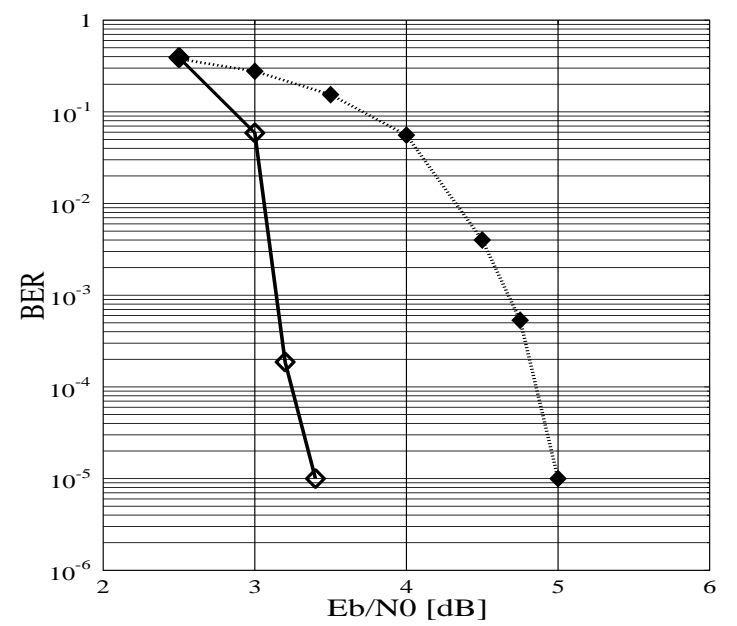

The serially concatenated LT-GLDPC, Approx-log MAP, Scheme 1 The serially concatenated LT-GLDPC,Approx-log MAP, Scheme 2

Figure 7: The performance of the serially concatenated LT and G-LDPC code, using BPSK modulation for transmission over the BEC-AWGN channel, $P_{e}=0.1$

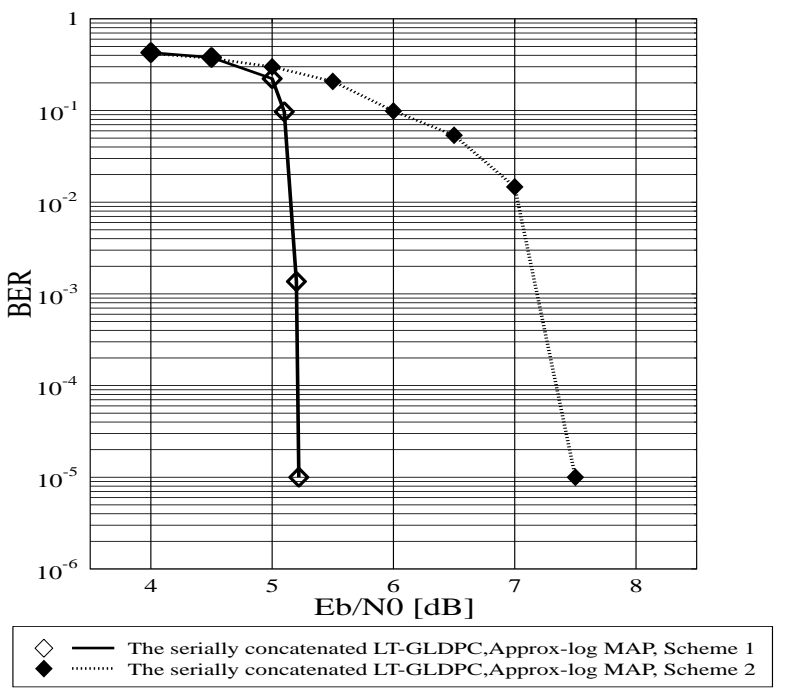

Figure 8: Performance of the serially concatenated LT and GLDPC code, using BPSK modulation for transmission over the BEC-Rayleigh channel, $P_{e}=0.1$ 


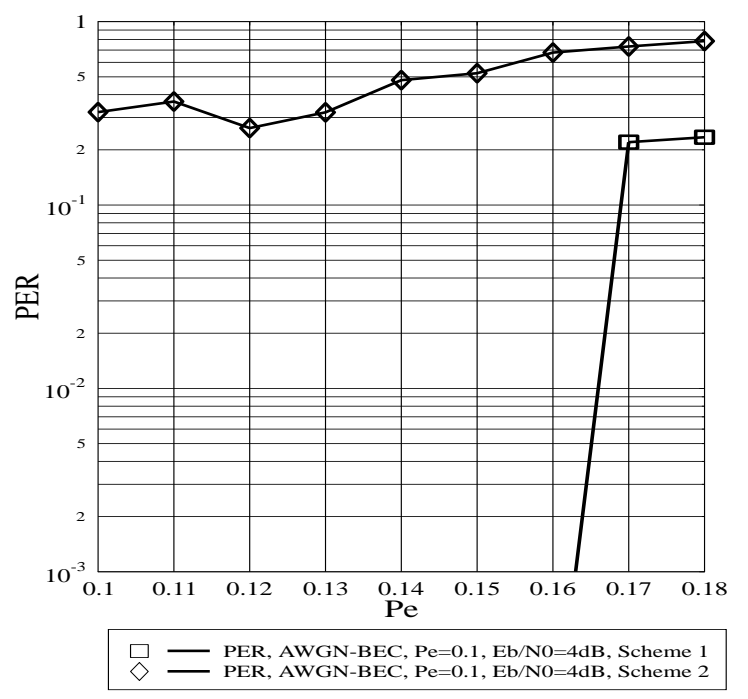

Figure 9: PER performance of the amalgamated LT-GLDPC Scheme 1 and Scheme 2 versus $P_{e}$ in AWGN-BEC channel, $E_{b} / N_{0}=4 \mathrm{~dB}$

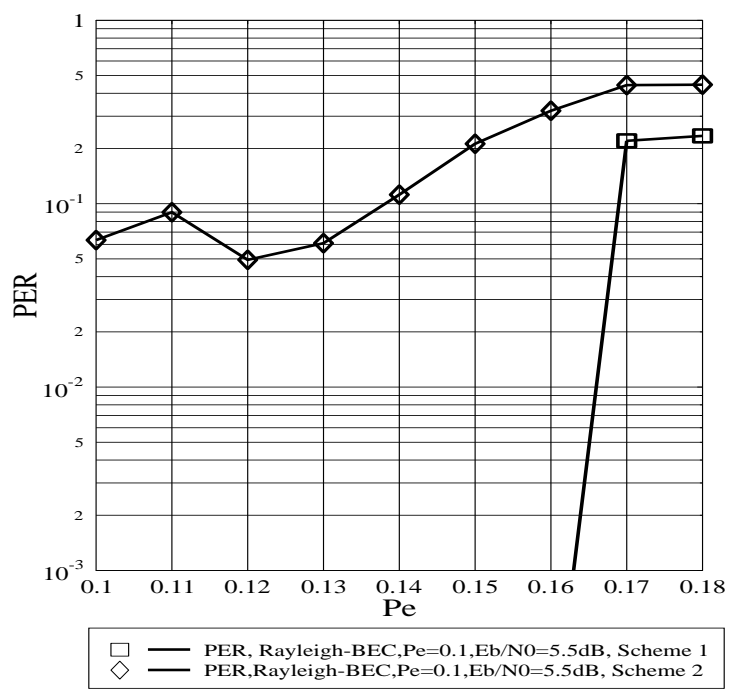

Figure 10: PER performance of the amalgamated LT-GLDPC Scheme 1 and Scheme 2 versus $P_{e}$ in Rayleigh-BEC channel, $E_{b} / N_{0}=5.5 \mathrm{~dB}$ 